\title{
Development of the Sleep and Wakefulness Rhythm in Preterm Infants Discharged from a Neonatal Care Unit ${ }^{1}$
}

\author{
MIEKO SHIMADA. MASAYA SEGAWA. MAKOTO HIGURASHI. AND HIROSHI AKAMATSU \\ Department of Waternal and (hild Health. School of Health Sciences, Faculty of Hedicine, The Lniversits of

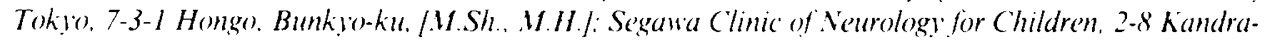 \\ surugadai. Chiyoda-ku, /W.Se.l: and Deparment of Newhorn and Premature . Medicine. Japanese Red (ross \\ Hedical ('enter. 4-1-22 Hiroo. Shibura-ku /H..4.J. Tokyo. Japan
}

\begin{abstract}
The purpose of this study was to investigate the effect of constant light in a neonatal care unit on the development of the sleep-and-wakefulness rhythm in preterm infants. Two groups of infants (57 preterm infants without other complications and 58 healthy term infants) were prospectively studied over infancy by a day-by-day plot method, by which sleep-and-wakefulness states were recorded at home for more than $14 \mathrm{~d}$ to compare developmental courses of the sleep-and-wakefulness rhythm between the two groups at corrected and postnatal ages. In the two groups, there were no significant differences in distribution of emergence of periodicity of sleep states and wakeful states, total sleep time, nocturnal sleep time, diurnal sleep time, longest sustained sleep period, and longest sustained wakeful period at the same corrected ages. Moreover, the SD of the time of onset of the longest sustained sleep period of each subject diminished with increase in postconceptional weeks. The results suggest that the development of the sleep-and-wakefulness rhythm in preterm infants is not necessarily retarded if they are discharged from the neonatal care unit under constant light before an infant's innate biologic clock is mature enough to respond to an environmental cycle; rather it depends on their corrected ages. (Pediatr Res 33: 159-163, 1993)
\end{abstract}

\section{Abbreviations}

SCN, suprachiasmatic nuclei

TST, total sleep time

NST, nocturnal sleep time

DST, diurnal sleep time

LSP, longest sustained sleep period

LWP, longest sustained wakeful period

Recent advances in perinatal medicine and care have led to an increase in survival rate of premature infants. As a result, premature infants, particularly those with very low birth weight, are treated under constant light for a long time in a neonatal care unit (1).

The generation and entrainment (synchronization to a 24-h cycle) of circadian rhythms such as sleep-and-wakefulness rhythm are mediated by the SCN of anterior hypothalamus in

Received April 27. 1992: accepted October 6. 1992.

Correspondence: Mieko Shimada. Department of Maternal and Child Health. School of Health Sciences, Faculty of Medicine, University of Tokyo. Hongo 7-31. Bunkyou-ku, Tokyo, 113 Japan.

1 This study was presented at the 17th annual meeting of the Japanese Society of Sleep Research with additional data of low-birth-weight term infants. mammals (2). For development of the circadian rhythm, it is necessary to entrain the innate biologic clock to environmental cycles, mostly to the light-dark cycle in a certain postnatal period (3). Therefore, an adequate nursing environment in early infancy, particularly the light-dark cycle, is essential for development of the circadian rhythm of sleep and wakefulness. It is suggested by animal studies that the quality of the environmental cycle during a certain period after birth regulates the circadian rhythm of sleep and other functions of infants (4). This raises the question of whether exposure to light with constant intensity in the neonatal care unit for a long period affects the development of the circadian rhythm in premature infants.

However, there have been few studies on the influence of the light-dark cycle on the development of the sleep-and-wakefulness cycle in premature human infants $(5,6)$. Lacking also are studies on the long-term effects of constant light in the neonatal care unit on the development of circadian rhythm. Therefore, this study was undertaken to determine whether preterm infants discharged from the neonatal care unit showed any retardation in development of the circadian rhythm of sleep and wakefulness throughout infancy.

\section{SUBJECTS AND METHODS}

Subjects. Of 423 premature infants who were admitted to the neonatal care unit at Japanese Red Cross Medical Center in Tokyo from February 1989 to September 1990, 123 preterm (born earlier than 37 gestational wk) infants weighing 1200-2300 $\mathrm{g}$ at birth who showed good therapeutic progress were all subjected to this study. Their gestational wk ranged from 28 to 36 . These infants did not manifest any complications that are known to affect sleep states, such as theophylline treatment (7), respiratory distress (8), heart disease (9), neurologic disorder (including brain disease) (10), history of neonatal distress (10), or thyroid disease (11). Infants were excluded from this study if they manifested any of these complications during hospitalization or any time thereafter. Of the 123 infants, 57 preterm infants whose parents gave informed consent were studied. As a normal control. 278 of 4614 healthy term (born 37 gestational wk onward) infants born weighing over $2500 \mathrm{~g}$ in the same medical center were randomly selected. Of these healthy term infants born at 37 to 42 gestational wk from September 1989 to September 1991. 58 infants whose parents gave informed consent were studied. On the 2 nd $d$ of life, the healthy term infants were transferred to their mothers in the postpartum ward where the lights were turned off during the night. The ages of the infants ranged from 0 to $12 \mathrm{mo}$.

Method. A sleep diagram and a questionnaire to determine the infants' environment (e.g. light-dark conditions at home, parental interventions), night crying, and night waking were 
handed at discharge from the neonatal care unit or sent to mothers of the 123 preterm infants from April to September 1990 and to mothers of the 278 control infants from September 1990 to September 1991. Mothers recorded their infants' daily activities on a sleep diagram for $14 \mathrm{~d}$ at minimum and for 12 corrected mo at maximum (for $12 \mathrm{wk}$ in median) by the day-byday plot method. With this method, the observer or subject records the time of onset of daily activities such as sleeping, waking, eating, or feeding on a successive 24-h diagram. Reliability of this method has been confirmed. Spangler (12) assessed the results of chronologic records of infants' sleep (so-called dayby-day plot method) recorded by mothers and those of the infants' adrenocortical activity, the circadian rhythm of which is related to the sleep-wake cycle in adults. The findings revealed that the 24-h sleep-wake cycle paralleled the circadian rhythm pattern in adrenocortical activity. Matsuoka et al. (13) videotaped the activities of two infants over seven nights at home while their mothers were sleeping. These mothers recorded the infants' activities by the day-by-day plot method on a sleep diagram. The time lag between the actual video-recorded sleep and the time recorded by the mothers was less than $15 \mathrm{~min}$ (13). These data assured that a time lag in a sleep diagram has a negligible effect on the analysis based on data recorded at intervals of $30 \mathrm{~min}$.

Data such as gestational age, birth weight, sex, clinical diagnosis, quality of intensive care, duration of phototherapy, and the number of days of hospitalization of these subjects were obtained from the clinical charts of the hospital. The gestational age of each infant was determined from the obstetrical estimated date of conception, which was based either on the date of the mother's last menstrual period or on ultrasound examination. The design of this study was accepted by the hospital ethics committee.

Light intensity was measured for $3 \mathrm{~d}$ every $4 \mathrm{~h}$ in this neonatal care unit and in the common home of a preterm infant by using a light meter (Tokyo Photo Electric Co., Tokyo, Japan).

Data analysis. Data of sleep and wakefulness behavior in the sleep diagram were numerically coded at intervals of $30 \mathrm{~min}$. Those data that were recorded in any physically abnormal condition such as a febrile episode or insufficient data were excluded from analysis. Only subjects with valid data days for more than $13 \mathrm{~d}$ were analyzed. The valid data were obtained for 57 preterm infants $(3808 \mathrm{~d})$ and 58 healthy term infants $(3278 \mathrm{~d})$. Based on increasing developmental maturity of the sleep-and-wakefulness rhythm, these data were analyzed for the following four parameters: 1) existence of periodicity of sleep and wakefulness (periodic appearance of sleep time and wakeful time); 2) TST (increase in wakeful time); 3) duration of diurnal wakeful time and NST (entrainment of sleep-and-wakefulness rhythm to a 24$\mathrm{h}$ cycle); and 4) duration of the LSP and the LSW (prolongation of sustained sleep and wakefulness).

Statistical comparisons of the difference of sleep time between preterm and healthy term infants were made by the comparison of the means (unpaired $t$ test). The rate of TST to $24 \mathrm{~h}$, NST to TST, DST to NST, LSP to TST, and LWP to total wakeful time were compared by comparison of the proportions (unpaired $t$ test). The frequency of light condition, etc., at home in the two groups was compared by $\chi^{2}$ test. The existence of periodicity was tested by two-way analysis of variance (14) for each individual infant every $14 \mathrm{~d}$. A significant $F$ value $(p<0.01)$ from the analysis of variance table for the column is interpreted as the existence of periodicity, where the column-factor is time in a day and the row-factor is day (15). The time for periodicity of sleep and wakefulness to emerge was determined by the weeks when the significant $F$ value was shown first. The distribution of the time taken for the periodicity to emerge in both groups was statistically compared by the generalized Wilcoxon test.

Furthermore, the mean of the time of onset of LSP was calculated for each individual infant every $14 \mathrm{~d}$ to determine when the LSP appeared in $24 \mathrm{~h}$, and the SD of the time of onset of LSP was also calculated as the mean to determine whether the LSP appeared regularly.

\section{RESULTS}

Informed consent was obtained for 57 of 123 preterm infants (46.3\%) and 60 of 278 healthy term infants. However, in the latter group, two were invalidated, resulting in 58 subjects $(20.9 \%)$. The number of subjects is shown in Table 1. The demographic and clinical characteristics of the subjects are shown in Table 2.

The light intensity in this neonatal care unit ranges from 420 to 500 lux throughout the day. The intensity in the home was

Table 1. Number of subjects by months

\begin{tabular}{cccccc}
\hline $\begin{array}{c}\text { Corrected } \\
\text { age }\end{array}$ & $\begin{array}{c}\text { Preterm } \\
\text { infants }\end{array}$ & $\begin{array}{c}\text { Healthy } \\
\text { term } \\
\text { infants }\end{array}$ & $\begin{array}{c}\text { Postnatal } \\
\text { mo }\end{array}$ & $\begin{array}{c}\text { Preterm } \\
\text { infants }\end{array}$ & $\begin{array}{c}\text { Healthy } \\
\text { term } \\
\text { infants }\end{array}$ \\
\hline $35-39 \mathrm{wk}$ & 14 & 7 & & & \\
$0 \mathrm{mo}$ & 23 & 25 & 0 & 11 & 25 \\
$1 \mathrm{mo}$ & 20 & 26 & 1 & 23 & 25 \\
$2 \mathrm{mo}$ & 18 & 26 & 2 & 20 & 26 \\
$3 \mathrm{mo}$ & 19 & 25 & 3 & 18 & 24 \\
$4 \mathrm{mo}$ & 19 & 21 & 4 & 18 & 21 \\
$5 \mathrm{mo}$ & 16 & 10 & 5 & 19 & 10 \\
$6 \mathrm{mo}$ & 16 & 8 & 6 & 19 & 8 \\
$7 \mathrm{mo}$ & 13 & 6 & 7 & 16 & 6 \\
$8 \mathrm{mo}$ & 6 & 6 & 8 & 15 & 6 \\
$9 \mathrm{mo}$ & 6 & 6 & 9 & 7 & 6 \\
$10 \mathrm{mo}$ & 8 & 5 & 10 & 7 & 5 \\
$11 \mathrm{mo}$ & 7 & 6 & 11 & 9 & 6 \\
$12 \mathrm{mo}$ & 1 & 4 & 12 & 5 & 3 \\
\hline
\end{tabular}

Table 2. Demographic characteristics of subjects

\begin{tabular}{|c|c|c|}
\hline & $\begin{array}{l}\text { Preterm infant } \\
\quad(n=57)\end{array}$ & $\begin{array}{c}\text { Healthy term } \\
\text { infant } \\
(n=58)\end{array}$ \\
\hline \multicolumn{3}{|l|}{ Sex } \\
\hline No. of male & 30 & 23 \\
\hline No. of female & 27 & 35 \\
\hline \multicolumn{3}{|l|}{ Birth order } \\
\hline No. of first born & 30 & 44 \\
\hline No. of subsequent born & 27 & 14 \\
\hline \multicolumn{3}{|l|}{ Gestational age at birth (wk) } \\
\hline Mean (SD) & $33.6(1.8)$ & $39.4(1.2)$ \\
\hline Range & $28-36$ & $37-42$ \\
\hline \multicolumn{3}{|l|}{ Birth weight $(\mathrm{g})$} \\
\hline Mean (SD) & $1966(240)$ & $3037(306)$ \\
\hline Range & $1268-2284$ & $2526-3676$ \\
\hline No. small for gestational date & 4 & 0 \\
\hline $\begin{array}{l}\text { No. appropriate for gestational } \\
\text { date }\end{array}$ & 53 & 58 \\
\hline \multicolumn{3}{|l|}{$\begin{array}{l}\text { Days of hospitalization in neona- } \\
\text { tal care unit (d) }\end{array}$} \\
\hline Mean (SD) & $32.1(12.8)$ & $2.0(0)$ \\
\hline Range & $11-69$ & 2 \\
\hline \multicolumn{3}{|l|}{$\begin{array}{l}\text { Corrected age at discharge from } \\
\text { neonatal care unit (wk) }\end{array}$} \\
\hline Mean (SD) & $38.5(1.4)$ & $40.4(1.2)$ \\
\hline Range & $36-43$ & $37-42$ \\
\hline No. with neonatal complications & 48 & 2 \\
\hline Hypoglycemia & 4 & 0 \\
\hline Hypocalcemia & 1 & 0 \\
\hline Hyperbilirubinemia & 23 & 2 \\
\hline $\begin{array}{l}\text { Transient tachypnea of new- } \\
\text { born }\end{array}$ & 14 & 0 \\
\hline Infection & 3 & 0 \\
\hline Other & 7 & 0 \\
\hline
\end{tabular}


3000 lux at fine daytime (650 lux through a curtain). 1200 to 2000 lux at cloudy daytime, and, at night, 100 to 200 lux in full light, 2 to 9 lux in dim light. The light condition during night sleep at home was five, 16,33, and three infants with lighting on, with dim light, with light off, and unknown, respectively, in the preterm infant group, and five, 19, 26, and eight infants, respectively, in the healthy term infant group. This frequency was not significantly different in the two groups. There was no significant difference in the frequency of self-demand feeding or regular-time feeding during the first 4 mo in the two groups. The mean bedtime of these parents was not significantly different between the two groups.

The cumulative frequency distribution of the time taken for periodicity of sleep and wakefulness to emerge was significantly different $(p<0.01)$ in the postnatal weeks (weeks after birth) between the two groups, with the preterm infants lagging behind the healthy term infants. Periodicity of sleep and wakefulness had emerged in $50 \%$ of the preterm infants by 11 postnatal wk and in $50 \%$ of the healthy term infants by 5 postnatal wk. However, this cumulative frequency distribution was not significantly different in postconceptional weeks (gestational weeks plus postnatal weeks) between the two groups (Fig. 1). The periodicity had emerged in $50 \%$ of either group by 44 postconceptional wk. There were some who showed periodicity of sleep and wakefulness occurring shortly after birth, at 39 postconceptional wk or 3 postnatal wk in preterm infants and at 40 postconceptional weeks or 2 postnatal weeks in healthy infants.

TST gradually decreased with age in both preterm infants and healthy term infants. In preterm infants, it was significantly longer than in healthy term infants at the postnatal ages of 0 and $5-6 \mathrm{mo}(p<0.01)$, and 1 and $7 \mathrm{mo}(p<0.05)$. TST in preterm infants was longer than in healthy term infants at -1 corrected month ( 36 to 39 postconceptional wk). However, at the other corrected months (months after expected date of birth), there was no significant difference between the two groups (Fig. 2).

NST gradually increased with age in both groups (Fig. 3). NST in preterm infants was significantly longer than in healthy term infants at the postnatal age of $0 \mathrm{mo}(p<0.01)$ and $5 \mathrm{mo}(p<$ $0.05)$. Although NST in preterm infants was longer than in healthy term infants at the corrected ages of -1 and 4 mo $(p<$ $0.01)$, and 3 mo $(p<0.05)$ (Fig. 3), the rate of NST to TST showed no significant difference between the two groups at any corrected month.

DST decreased rapidly up to 5 corrected months. In preterm infants, it was significantly longer than in healthy term infants at the postnatal ages of 0 and 5 mo $(p<0.05)$. DST in preterm infants was longer than in healthy term infants at -1 corrected month (36 to 39 postconceptional wk) $(p<0.05)$. However, at the other corrected months, there was no significant difference between the two groups (Fig. 4). The ratio of DST to NST showed no significant difference between the two groups at any corrected month. This means that DST in preterm infants was not different in the two groups in corrected months.

The LSP in $24 \mathrm{~h}$ increased gradually with age. In healthy term infants, it was significantly longer than in preterm infants at postnatal ages of 1 and $2 \mathrm{mo}(p<0.01)$, and $3 \mathrm{mo}(p<0.05)$. LSP in preterm infants was longer than in healthy term infants at corrected ages of $5 \mathrm{mo}(p<0.01)$, and 4 and 11 mo $(p<$ 0.05 ) (Fig. 5). However, the rate of LSP to TSP showed no significant difference between the two groups.

The LWP increased slightly with age from approximately 3.7 $\mathrm{h}$ at 0 corrected month to $5.8 \mathrm{~h}$ at 10 corrected months. In healthy term infants, it was longer than in preterm infants at 0 postnatal month $(p<0.05)$. However, at the other postnatal months and all corrected months, there was no significant difference between the two groups.

The means of the time of onset of LSP in the individual subjects were between 2100 and $0100 \mathrm{~h}$ from just after birth in all preterm and healthy term infants. SD of the time of onset of LSP in the individual preterm infants showed a negative correlation with postconceptional weeks (Spearman's $r=-0.60, p=$ 0.001 ) (Fig. 6), the same as healthy term infants (Spearman's $r$ $=-0.57, p=0.0001)$. It decreased with the number of postconceptional weeks, and had diminished rapidly (Pearson's $r=$ $-0.76, p<0.000)$ by 60 postconceptional wk $(5$ corrected months).

\section{DISCUSSION}

It has been established that sleep problems and delay in sleepand-wakefulness rhythm are more common in premature infants

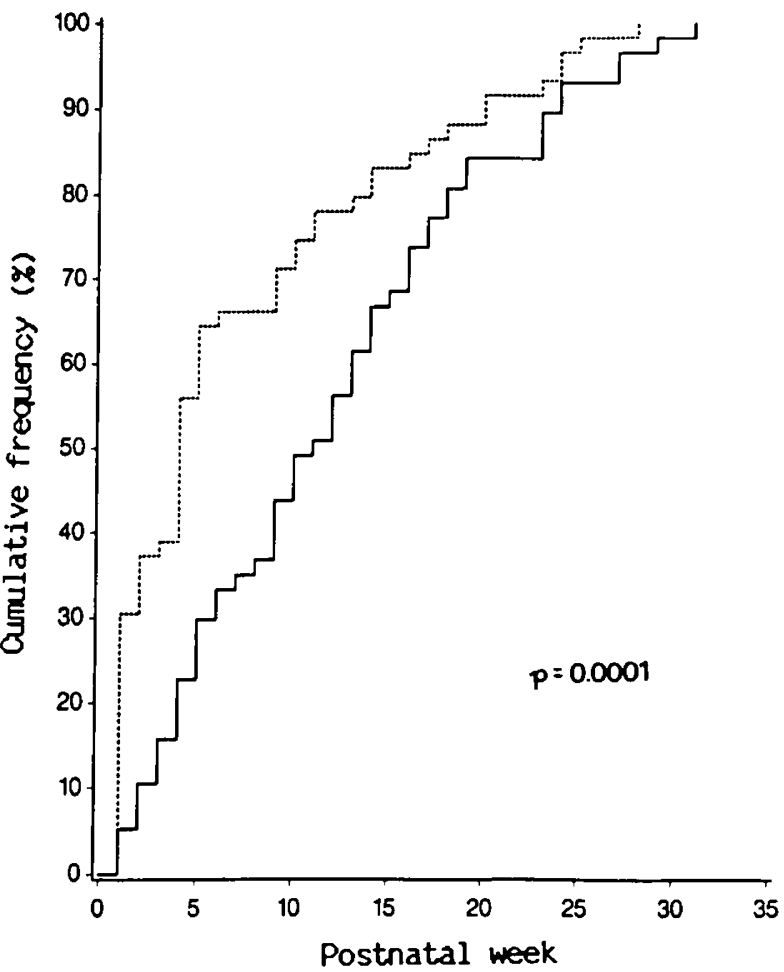

Fig. 1. Distribution of the time of emergence of periodicity. 


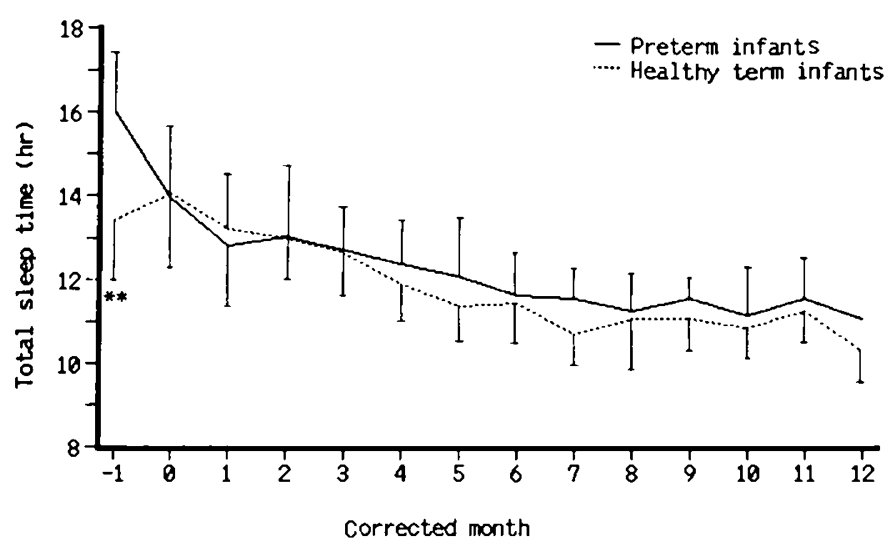

Fig. 2. TST in preterm and healthy term infants. ${ }^{* *}, p<0.01$.

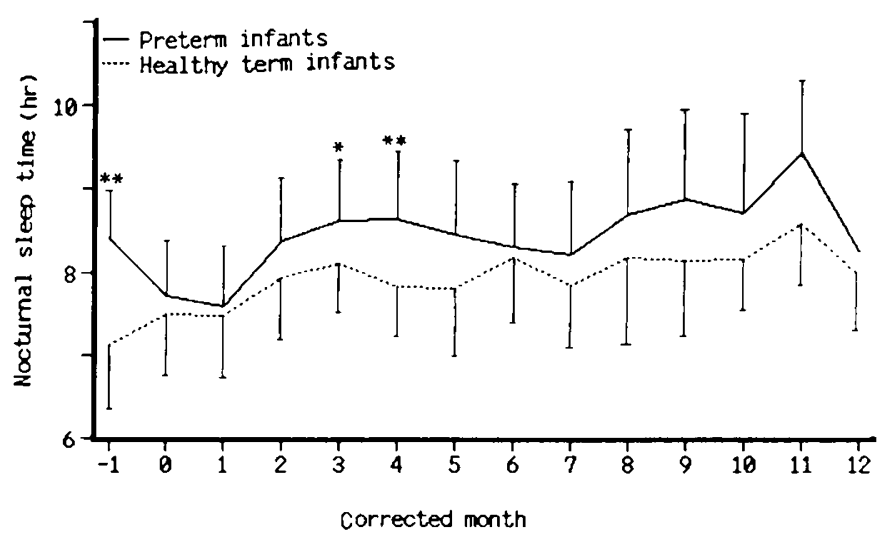

Fig. 3. NST in preterm and healthy term infants. ${ }^{*}, p<0.05:{ }^{* *}, p<$ 0.01

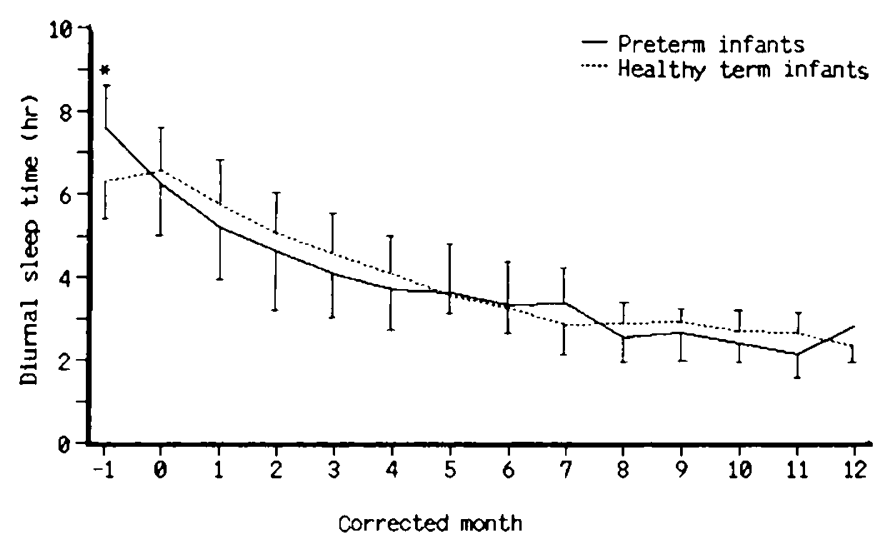

Fig. 4. DST in preterm and healthỵ term infants. ${ }^{*}, p<0.05$.

than in healthy term infants $(5,16)$. In this study, however, the ratios of NST to TST, of DST to NST, of LSP to TST, and LWP to total wakeful time showed no significant difference between the two groups at any corrected months. TST showed no significant difference between the two groups at the corrected ages except for -1 corrected month ( 36 to 39 postconceptional wk). Moreover, there was no significant difference in cumulative frequency distribution of the time taken for periodicity to emerge between the two groups at corrected ages. Therefore, it is suggested that the development of sleep-and-wakefulness rhythm in preterm infants is not necessarily retarded at corrected ages.

We focused on the LSP and investigated whether it would appear regularly over $24 \mathrm{~h}$ by determining the SD of the time of onset of LSP of each individual subject. The LSP is assumed to form the main part of sleep, as the maturation of the CNS contributes to the prolongation of sleep and the wakeful state (17). It is also assumed that the sleep rhythm of a $24-\mathrm{h}$ cycle is established if LSP appears in a certain nocturnal period. This method is thought to be an appropriate and simple analysis for observing the entrainment of sleep-and-wakefulness rhythm to a 24-h cycle in the mass. In this study, LSP emerged in a nocturnal period, and the individual SD of the time of onset of LSP diminished with increase in the postconceptional weeks. The onset time was gradually concentrated on each individual at certain times in both preterm and healthy term infants. These results indicate that the sleep-and-wakefulness rhythm in both preterm and healthy term infants is entrained, and they suggest that the development of a circadian rhythm of sleep depends on the postconceptional week corresponding to the development of the nervous system. It is considered that the development of the circadian rhythm of sleep is related to an inner factor, i.e. the innate biologic clock needs to mature to a level sufficient to be able to receive environmental cycles before infants can synchronize their behavior with an environmental cycle, such as being put to bed at the same time.

It is speculated that the 24-h environmental cycle, which acts via the retinohypothalamic pathway to entrain the circadian

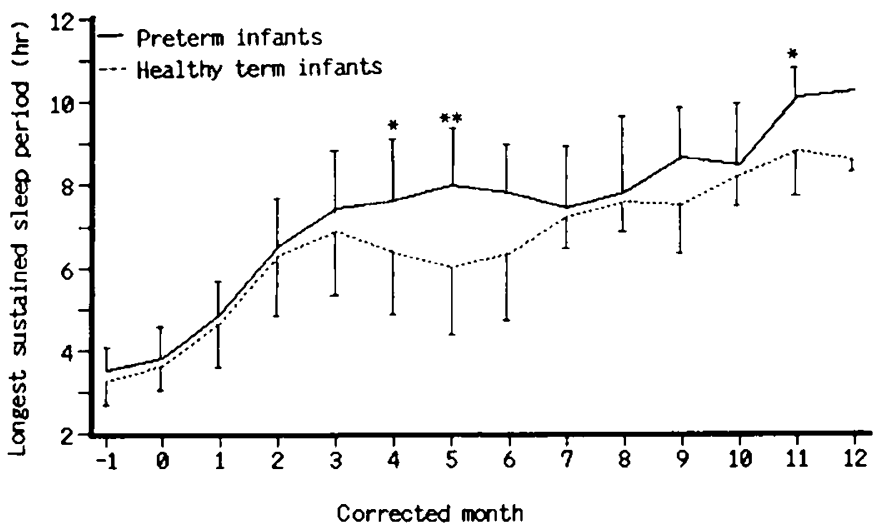

Fig. 5. LSP in preterm and healthy term infants. ${ }^{*}, p<0.05:{ }^{* *}, p<$ 0.01 .

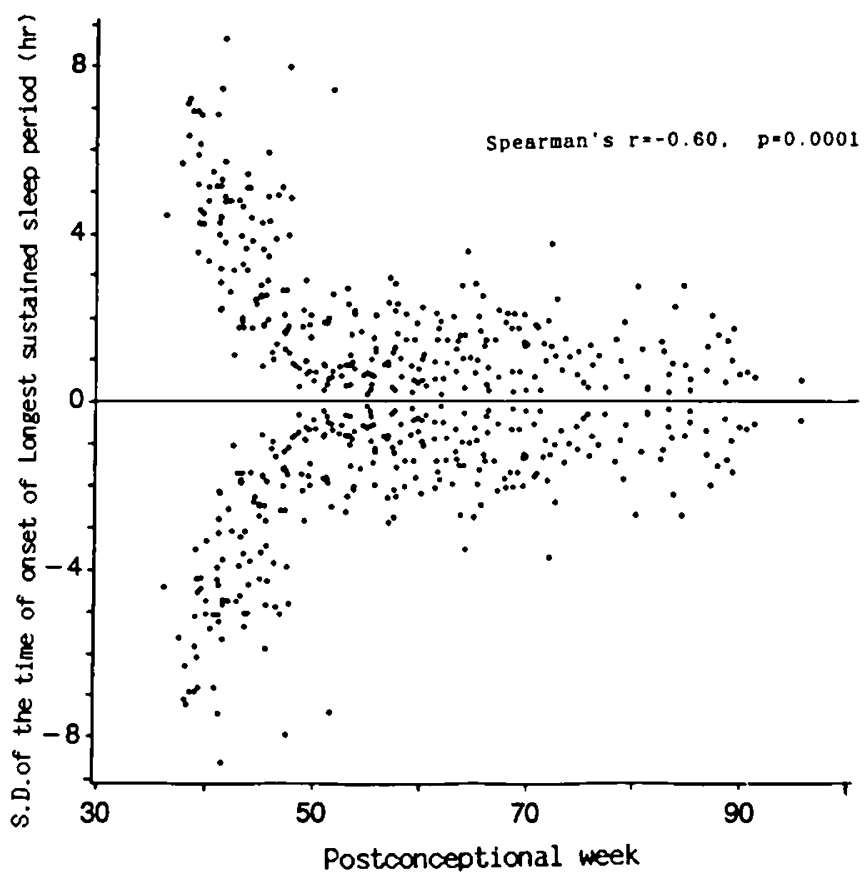

Fig. 6. Variance of the time of onset of LSP in preterm infants. SD $=0$ is based on the individual mean of the time of onset of LSP every $14 \mathrm{~d}$ in each individual preterm infant: $i$. $e^{\prime}$. the individual mean value. which ranges from 2100 to $0010 \mathrm{~h}$, is arranged for $\mathrm{SD}=0$. Each point on the figure shows the SD of the time of onset LSP every $14 \mathrm{~d}$ in each preterm infant. 
clock in the SCN, has an effect only on the biologic clock matured to a certain level but plays no essential role as a time cue to form circadian rhythms for the immature brain of infants. It is also suggested that an environment without a light-dark cycle in a neonatal care unit has little influence on the development of the sleep-and-wakefulness rhythm until development onset of retinohypothalamic tract at approximately 40 postconceptional wk, the approximate age of the subjects in this study when they were discharged from the neonatal care unit. Myelination around optic nerves fibers occurs after a full-term (40-wk) gestation in preterm and term human infants (18), and it is suggested that afferent retinal fibers in term rats first reach the SCN on the 2 nd or 3 rd postnatal $\mathrm{d}$ and invade the SCN by the $3 \mathrm{rd} \mathrm{d}$ before they have formed synapses in the SCN (19). After the neurologic development to a certain maturational level, the light-dark cyclic environment is rather important for establishing the sleep-and-wakefulness rhythm.

The time of onset of LSP was predominantly between 2100 and $0100 \mathrm{~h}$ in all infants in this study. This probably reflects the 24-h rhythm of the pregnant mother who went to bed around this time, because it has been suggested that circadian rhythm in infants is formed in utero based on the mother's circadian rhythm (20). Human (21) and animal (22) observations suggest that abnormality of an infant's circadian rhythm can be corrected if he or she is reared under appropriate 24 -h cycles within a certain period of life. The following observations may be considered: the preterm infants kept the rhythm based on the mothers' rhythm even in the neonatal care unit under constant light, the rhythm was corrected after discharge by an altered environment, and preterm infants were able to establish the same sleep-and-wakefulness rhythm as healthy term infants. These observations suggest that maturational level of the nervous system is crucial in forming the circadian rhythm of sleep by external factors.

There have been some studies on the end point of the critical period of formation of circadian rhythm $(4,22)$. However, its starting point, from which an innate biologic clock of an infant is affected by external factors, has not been studied. In the future, we will try to determine the starting point when the innate biologic clock matures to the level affected by an environmental cycle such as constant light. A study on whether the same development of circadian rhythm of body temperature, hormones, and other factors is established in preterm infants in a neonatal care unit is also necessary.

The maturation of the CNS is reported to play an important role in the development of sleep (17); nevertheless, there is less elucidation of effect of constant light on the subsequent development in preterm infants, in whom there was a risk of prematurity. We are proceeding with a cohort study on the long-term development of behavior and circadian rhythm of sleep and hormones in the preterm infants.

In conclusion, there is not necessarily any developmental retardation of the sleep-and-wakefulness rhythm in preterm infants at corrected ages. The development of the sleep-and-wakefulness rhythm depends on the infants' postconceptional weeks. It is suggested that a neonatal care unit under constant light has little influence on the development of the sleep-and-wakefulness rhythm if a preterm infant is discharged from the unit before the infant's innate biologic clock and the retinohypothalamic path- way are mature enough to respond to an environmental cycle. The care of infants in a light-dark cyclic environment seems to be essential to the development of a sleep-and-wakefulness rhythm after maturation to a certain level of the infant's innate biologic clock and retinohypothalamic pathway.

Acknowledgment. The authors thank Professor Yasuo Ohashi of the Department of Epidemiology and Biostatistics of the University of Tokyo, for advising us regarding statistical analysis.

\section{REFERENCES}

1. Maternal and Child Health Division, Children and Families Bureau, Ministry of Health and Welfare 1991 Maternal and Child Health Statistics of Japan. Tokyo, Japan

2. Moor RY 1983 Organization and function of central nervous system circadian oscillator: the suprachiasmatic hypothalamic nucleus. Fed Proc 42:27832789

3. Moor RY, Card JP 1985 Visual pathways and entrainment of circadian rhythms. Ann NY Acad Sci 453:123-133

4. Sasaki Y, Murakami N, Takahashi K 1984 Critical period for the entrainments of the circadian rhythm in blinded pups by dams. Physiol Behav 33:105109

5. Mann NP, Haddow R, Stokes L, Goodley S, Rutter N 1986 Effect of night and day on preterm infants in a newborn nursery. Br Med J 293:1265-1269

6. McMillen IC, Kok JSM, Adamson TM, Deayton JM, Nowak R 1990 Development of circadian sleep-wake rhythms in preterm and full-term infants. Pediatr Res 29:381-384

7. Thoman EB, Davis DH, Raye JR, Phillipps AF, Rowe JC, Denenberg VH 1985 Theophilline affects sleep-wake state development in premature infants. Neuropediatrics 16:13-18

8. McGinty DJ 1985 The physiological equilibrium and the control of sleep states. In: McGinty DJ, Druker-Colin R, Morrison A, Pameggian PL (eds) Brain and Mechanisms of Sleep. Raven Press, New York, pp 360-384

9. Yasuma F, Nomura H, Hayashi H, Okada T, Tuzuki M 1989 Breathing abnormalities during sleep in patients with chronic heart failure. Jpn Circulation J 53:1506-1510

10. Okawa M, Takahashi K Sasaki H 1986 Disturbance of circadian rhythm in severely brain-damaged patients correlated with CT findings. J Neurol 223:274-282

11. Kales A, Heuser G, Jacobson A, Kales JD, Hanley J, Zweizig JR, Paulson MJ 1967 All night sleep studies in hypothyroid patients, before and after treatment. J Clin Endocrinol 27:1593-1599

12. Spangler G 1991 The emergence of adrenocortical circadian function in newborn and infants and its relationship to sleep. feeding and maternal adrenocortical activity. Early Hum Dev 25:197-208

13. Matsuoka M, Segawa M. Higurashi M 1991 The development of sleep/ wakefulness cycle in early infancy and its relation to feeding habit. Tohoku J Exp Med 165:147-154

14. Enlight JT 1965 The search for rhythmicity in biological time-series. J Theor Biol 8:426-468

15. Wever RA 1979 The analyses of circadian rhythm. In: Wever RA (ed) The Circadian System of Man. Springer Verlag. New York, pp 17-24

16. Sammons WAH, Lewis JM 1985 Sleep. In: Sammons WAH, Lewis JM (eds) Premature Babies: A Different Beginning. The CV Mosby Company, St. Louis, pp 223-233

17. Parmelee Jr AH, Stern E 1972 Development of states in infants. In: Clementte CD, Purpur DP. Mayer FE (eds) Sleep and the Maturing Nervous System. Academic Press, New York, pp 199-228

18. Magoon EH, Robb RM 1981 Development of myelin in human optic nerve and tract. Arch Ophthalmol 99:655-659

19. Stanfield B, Cowan WM 1976 Evidence for a change in retinohypothalamic projectin in the rat following early removal of one eye. Brain Res 104:129136

20. Reppert SM, Schwarz WJ 1983 Maternal coordination of the fetal clock in utero. Science 220:969-970

21. Matin du Pan R 1974 Some clinical applications of our knowledge of the evolution of the circadian rhythm in infants. In: Schevring LS. Halberg F Pauly JE (eds) Chronobiology. Igaku Syoin, Tokyo, pp 342-347

22. Takahashi K, Murakami N, Hayafuji C, Sasaki Y 1984 Further evidence that circadian rhythm of blinded rat is entrained by the nursing dam. Am J Physiol 246:359-363 